\title{
Magnetic composites as an effective technology for removal of radioactive cesium
}

\author{
S.-C. Jang ${ }^{1,2,3}$ - G. Y. Kim ${ }^{1}$ - S. B. Hong' ${ }^{2}$ H.-M. Yang ${ }^{2}$ - K.-W. Lee ${ }^{2}$ \\ J.-K. Moon ${ }^{2}$ B.-K. Seo ${ }^{2} \cdot$ Y. Cho ${ }^{4}$ Y. S. Huh ${ }^{3}$ C. Roh $^{1}$
}

Received: 12 November 2014/Revised: 10 April 2015/ Accepted: 6 July 2015/Published online: 18 August 2015

(C) Islamic Azad University (IAU) 2015

\begin{abstract}
Radioactive cesium $\left({ }^{137} \mathrm{Cs}\right)$ has seriously become a human concern owing to exposure from a nuclear accident release at a nuclear plant. Many efforts have focused at the removal of radioactive cesium and remediation of a contaminated environment. To meet these demands, an effective sorbent based on magnetic composites functionalized with synthetic clay minerals was demonstrated. This sorbent shows a high removal efficiency of contaminated water containing suspended sorbents at a level of $0.5 \mathrm{mg} \mathrm{ml}^{-1}\left[{ }^{137} \mathrm{Cs}\right.$ of 84.68 Becquerel (Bq) gram $\left.(\mathrm{g})^{-1}\right]$, decontaminated to $0.47 \mathrm{~Bq} \mathrm{~g}^{-1}$ (99.44\% removal efficiency) with just one treatment. The radioactive cesium is dramatically adsorbed into synthetic clay minerals. Subsequently, a rapid and easy sorbent separation from the radioactive cesium solution occurs after treatment using a magnetic field. Thus, a magnetic
\end{abstract}

C. Roh and Y. S. Huh have contributed equally to this work as cocorresponding authors.

\section{Roh}

chroh@kaeri.re.kr

1 Division of Biotechnology, Advanced Radiation Technology Institute, Korea Atomic Energy Research Institute, 1266 Shinjeong-dong, Jeongeup-si, Jeolabuk-do 580-185, Republic of Korea

2 Decontamination and Decommissioning Research Division, Korea Atomic Energy Research Institute, 989-111 Daedukdaero, Yuseong, Daejeon 305-353, Republic of Korea

3 Department of Biological Engineering, Biohybrid Systems Research Center (BSRC), Inha University, Inchon 402-751, Republic of Korea

4 Department of Environmental Engineering, Daejeon University, Daejeon 300-716, Republic of Korea sodium-phlogopite sorbent can offer high potential for in situ remediation.

Keywords Magnetic composites - Remediation Cesium $\cdot$ Sorbent $\cdot$ Removal

\section{Introduction}

Radioactive cesium $\left({ }^{137} \mathrm{Cs}\right)$ removal has become an emerging issue after the Fukushima Daiichi nuclear power plant accident owing to environmental contamination. And cesium bioavailability is strongly influenced by environmental properties such as potassium content, $\mathrm{pH}$, clay, and organic matter (Buesseler et al. 2012). Radioactive cesium based on its high radioactivity and relatively long half-life time (30.2 years) is a significant element of nuclear waste and nuclear fallout (Sangvanich et al. 2010). Cesium is water soluble and behaves similarly to potassium and sodium in a biological behavior profile. In addition, radioactive cesium can trigger negative health effects including carcinoma of the liver, kidney, bladder, renal function, cardiovascular disease, and gastrointestinal distress (Grignard et al. 2008; Guéguen et al. 2008; Souidi et al. 2006). Matrices as several different polymers, zeolitelike materials, clay minerals, biomass, and metal oxides have previously been investigated for Cs removal (Gupta and Dubey 2005; Mercer and Ames 1978). Among them, micas are ideal candidates for use as ion exchangers owing to their large layer charge and thermal stability. Phlogopite is an important and relatively common end-member composition of biotite. Phlogopite micas are found primarily in igneous rocks. In particular, Na-phlogopite excels at cesium removal (Stout et al. 2006). Recently, magnetic nanoparticles have been the focus of significant research 
because they possess attractive properties that could see potential use in tools including nanomaterials biomedicine, magnetic imaging, storage, sensors, and environmental remediation (Elliott and Zhang 2001; Gleich and Weizenecker 2005; Gupta and Gupta 2005; Hyeon 2003; Lu et al. 2004; Mahendran and Philip 2012). Among the many types of magnetic nanoparticles, iron oxide nanoparticles are especially interesting because they are both magnetic and catalytic. Iron oxide nanoparticles also have an advantage because they are easy to synthesize with size-dispersed products with high magnetic moments. Moreover, magnetic nanoparticles are a highly valuable substrate for the attachment of inorganic- and organic-containing catalysts. Iron oxide is generally considered biocompatible and is the only nanoparticle material to have been approved by the US Food and Drug Administration (Beveridge et al. 2011). In this study, a magnetic composite with Na-phlogopite as an effective technology for the removal of radioactive cesium was elucidated.

\section{Materials and methods}

\section{Chemicals}

All chemicals used in this work were of analytical reagent grade. Deionized water was used throughout the study. Iron(III) chloride hexahydrate $\left(\mathrm{FeCl}_{3} \cdot 6 \mathrm{H}_{2} \mathrm{O}\right)$, iron(II) chloride tetrahydrate $\left(\mathrm{FeCl}_{2} \cdot 4 \mathrm{H}_{2} \mathrm{O}\right)$, polyvinylpyrrolidone (PVP), potassium hexacyanoferrate(II) trihydrate $\left(\mathrm{K}_{3-}\right.$ $\left.\left[\mathrm{Fe}(\mathrm{CN})_{6}\right] \cdot 3 \mathrm{H}_{2} \mathrm{O}\right)$, a poly(diallyldimethylammonium chloride) (PDDA) solution (20 wt $\%$, MW 400,000-500,000), ammonium hydroxide $\left(\mathrm{NH}_{4} \mathrm{OH}\right)$, and hydrochloric acid ( $\mathrm{HCl})$ were purchased from Sigma-Aldrich Co., Ltd. In addition, cesium chloride $(\mathrm{CsCl})$ was purchased from Yakuri Co., Ltd.

\section{Preparation of poly(diallyldimethylammonium chloride) (PDDA)-coated iron oxide nanoparticles and Na-phlogopite}

A solution $(20 \mathrm{ml})$ containing iron(III) chloride hexahydrate $\left(\mathrm{FeCl}_{3} \cdot 6 \mathrm{H}_{2} \mathrm{O}\right)(2.16 \mathrm{~g})$, iron(II) chloride tetrahydrate $\left(\mathrm{FeCl}_{2} \cdot 4 \mathrm{H}_{2} \mathrm{O}\right)(0.8 \mathrm{~g})$, and poly(diallyldimethylammonium chloride) (PDDA) $(1.0 \%$, v/v) was deoxygenated by bubbling with nitrogen gas for $10 \mathrm{~min}$, followed by heating to $80{ }^{\circ} \mathrm{C}$. Subsequently, ammonium hydroxide $\left(\mathrm{NH}_{4} \mathrm{OH}\right)$
(28\%, $10 \mathrm{ml}$ ) was added rapidly to the heated solution, which was left to stir for another $1 \mathrm{~h}$. After cooling to room temperature, the formed PDDA-coated iron oxide $\left(\mathrm{Fe}_{3} \mathrm{O}_{4}\right)$ nanoparticles were isolated with the help of a magnet field and washed three times with deionized water. In addition, it was dried at room temperature. Finally, purified PDDAcoated iron oxide nanoparticles were obtained. For $\mathrm{Na}$ phlogopite, the large single crystals of phlogopite mica were broken into small pieces by hand followed by grinding in a household blender in the presence of deionized water. After grinding, the mica slurry was wet-sieved and the $<45-\mu \mathrm{m}$ size fraction was collected for use. Potassium was removed from the phlogopite mica flakes using the procedure described by Scott and Stout (Stout et al. 2006; Scott and Smith 1966). The $<45-\mu \mathrm{m}$ mica (5 g) was equilibrated with $100 \mathrm{ml}$ of a solution containing $1.0 \mathrm{M}$ sodium chloride $(\mathrm{NaCl})-0.2 \mathrm{~N}$ sodium tetraphenylborate- $0.01 \mathrm{M}$ disodium ethylenediaminetetraacetic acid (EDTA) for $168 \mathrm{~h}$ at room temperature. This procedure was repeated a total of three times to ensure the maximum removal of potassium from the interlayers of the mica. The samples were suction-filtered through Whatman 50 filter paper and washed repeatedly with a solution containing $40 \% 0.5 \mathrm{~N}$ sodium chloride-60\% acetone (v/ v) to remove the precipitated potassium tetraphenylborate from the sample. After the initial washings, the Na-phlogopite was washed with a large volume of deionized water under a vacuum to remove all entrained sodium chloride and allowed to air-dry.

\section{Preparation of magnetic Na-phlogopite}

PDDA-coated iron oxide $(1 \mathrm{~g})$ was dispersed in $10 \mathrm{ml}$ of deionized water, and $0.01 \mathrm{M} \mathrm{HCl}$ was added to adjust the $\mathrm{pH}$ to 6.0. Na-phlogopite $(3 \mathrm{~g})$, the $\mathrm{pH}$ of which ranges from 2.4 to 2.7 , was dispersed in $10 \mathrm{ml}$ of deionized water to which $0.01 \mathrm{M} \mathrm{NaOH}$ was added to adjust the $\mathrm{pH}$ to 6.0 . The slurry with PDDA-coated iron oxide and Na-phlogopite obtained was then mixed thoroughly at room temperature in a 50-ml polypropylene tube. Deionized water ( $25 \mathrm{ml}$ ) was added to this mixture, and a permanent magnet (1.4 tesla) was used to eliminate any excess Na-phlogopite that alone is not attracted by a magnetic field, and thus, we could magnetically separate the Na-phlogopite from the mixture of Na-phlogopite and non-Na-phlogopite. This purification step was repeated three times to obtain magnetic Na-phlogopite. 
Fig. 1 Schematic illustration of magnetic Na-phlogopite composites



PDDA
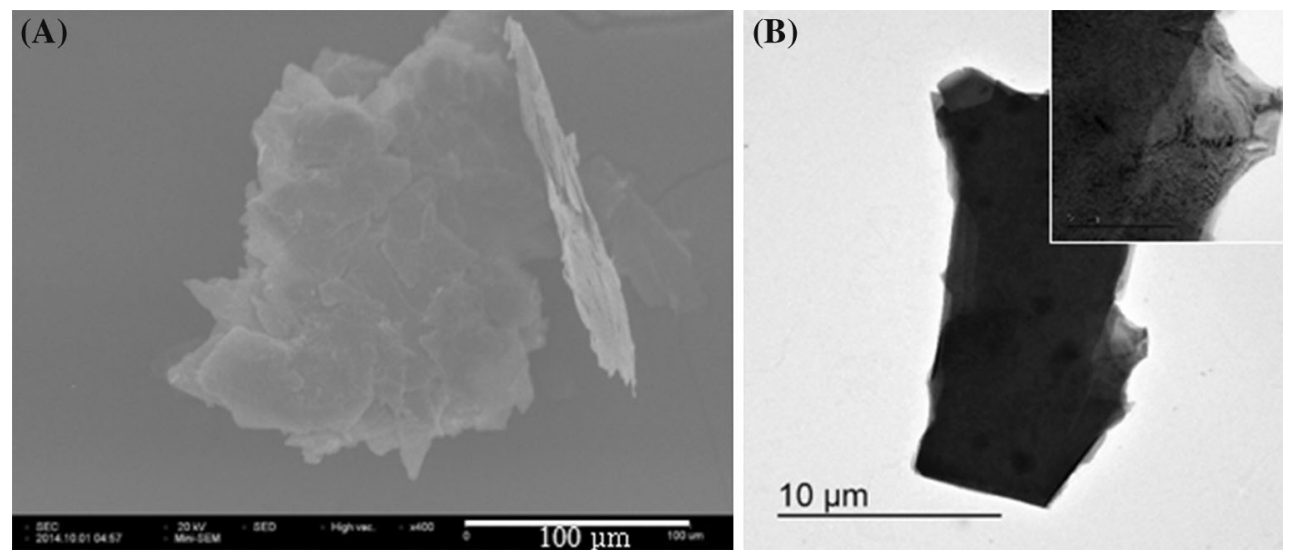

Fig. 2 Electron microscopy images a SEM of Na-phlogopite, b TEM of Na-phlogopite

\section{Characterizations and adsorption test}

The magnetic Na-phlogopite size and zeta potential were determined using a particle size analyzer (Malvern Zetasizer nano-ZS90, UK). Fourier-transform infrared (FT-IR) spectra of the magnetic Na-phlogopite and Na-phlogopite were recorded using a Thermo Scientific Nicolet iS5. The particle size and morphology of the nanoparticles were investigated using a scanning electron microscope (SEM) and transmission electron microscope (TEM) at an accelerating voltage of $300 \mathrm{keV}$ (Tecnai G2 F30, USA). And Cs sorption isotherm in batch operation was analyzed by ICPMS (Elandic II, PerkinElmer). The concentrations of the radioactive cesium $\left({ }^{137} \mathrm{Cs}\right)$ before and after treatment with the magnetic Na-phlogopite were measured using a highpurity germanium (HPGe) detector (Canberra, USA).

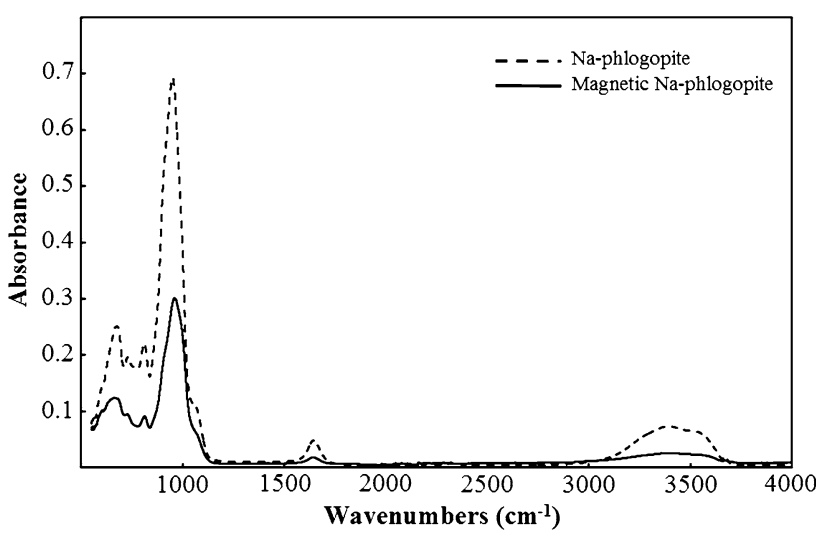

Fig. 3 FT-IR patterns of materials (a) Na-phlogopite, (b) magnetic Na-phlogopite 



Fig. 4 Magnetic separation process of Na-phlogopite. It was separated from radioactive cesium solution using a magnet

Table 1 Removal efficiency of the radioactive cesium $\left({ }^{137} \mathrm{Cs}\right)$ using magnetic Na-phlogopite

\begin{tabular}{|c|c|c|c|c|}
\hline Adsorbent concentration $\left(\mathrm{mg} \mathrm{ml}^{-1}\right)$ & Before treatment activity $\left(\mathrm{Bq} \mathrm{g}^{-1}\right)$ & After treatment activity $\left(\mathrm{Bq} \mathrm{g}^{-1}\right)$ & Removal efficiency (\%) & $\mathrm{DF}^{\mathrm{a}}$ \\
\hline 0.1 & 82.17 & 2.51 & 96.95 & 32.74 \\
\hline 0.5 & 84.68 & 0.47 & 99.44 & 180.17 \\
\hline 1 & 85.29 & 0.31 & 99.64 & 275.13 \\
\hline
\end{tabular}

${ }^{\mathrm{a}} \mathrm{DF}$ as a decontamination factor means ratio of activity prior to and after the decontamination of radioactively contaminated water

\section{Results and discussion}

The structure, morphology, and adsorption behavior of the Na-phlogopite magnetic composite were investigated. To coat Na-phlogopite on to the surface of PDDA-coated iron oxide, a method was shown using a negatively charged Naphlogopite (zeta potential $-70 \mathrm{mV}$ ) with a smaller amount of positively charged PDDA-coated iron oxide, and the surface area of the positively charged PDDA-coated iron oxide composites is entirely covered with negatively charged Na-phlogopite (Fig. 1). For sodium-phlogopite synthesis, phlogopite mica was equilibrated with $1.0 \mathrm{~N}$ sodium chloride- $-0.2 \mathrm{~N}$ sodium tetraphenylborate- $0.01 \mathrm{M}$ disodium ethylenediaminetetraacetic acid (EDTA) solutions at room temperature. Figure 2 shows the evaluation of a Na-phlogopite structure using SEM and TEM. As a shell, an evident strong resonance at $948 \mathrm{~cm}^{-1}$ is observed in the FT-IR spectrum of Na-phlogopite. Magnetic Na- phlogopite showed relatively low resonance owing to magnetic property (Fig. 3). As shown in Fig. 4, the separation of radioactive cesium $\left({ }^{137} \mathrm{Cs}\right)$ using an external magnet was rapidly achieved within $30 \mathrm{~s}$ in a batch experiment. The high ion exchange efficiency of Na-phlogopite in combination with the ease of the magnetic separation provided a facile way for the cesium decontamination. The magnetic composite showed high removal efficiency even in radioactive wastes at low concentrations of cesium. Finally, the magnetic Na-phlogopite can be easily recovered from radioactive cesium using a magnet. Table 1 shows the removal efficiency of radioactive cesium. Based on the concentration manner of the sorbent, radioactive cesium $\left({ }^{137} \mathrm{Cs}\right)$ of $82.17,84.68$, and $85.29 \mathrm{~Bq} \mathrm{~g}^{-1}$ was decontaminated to $2.51 \mathrm{~Bq} \mathrm{~g}^{-1}$ $\left(96.95 \%\right.$ at $\left.0.1 \mathrm{mg} \mathrm{ml}^{-1}\right), \quad 0.47 \mathrm{~Bq} \mathrm{~g}^{-1}(99.44 \%$ at $\left.0.5 \mathrm{mg} \mathrm{ml}^{-1}\right)$, and $0.31 \mathrm{~Bq} \mathrm{~g}^{-1}\left(99.64 \%\right.$ at $1.0 \mathrm{mg} \mathrm{ml}^{-1}$ ) with just one treatment. All of this means that radioactive 


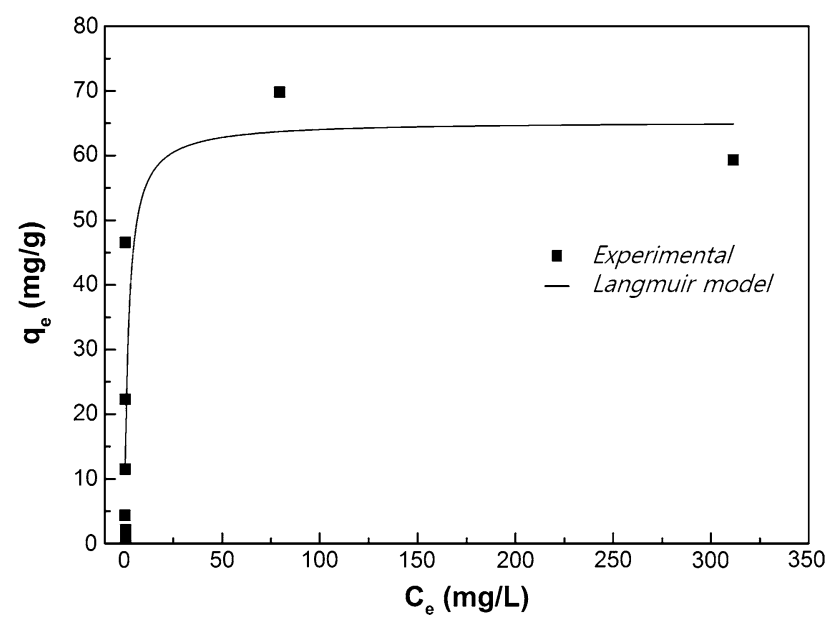

Fig. 5 Adsorption isotherm of the magnetic Na-phlogopite plotted between the equilibrium concentration of $\mathrm{Cs}$ in solution $\left(C_{\mathrm{e}}\right)$ and equilibrium concentration of Cs on adsorbent $\left(q_{\mathrm{e}}\right)$

cesium is dramatically adsorbed to Na-phlogopite in shells. Because of the high surface adsorption, the DF value as a decontamination factor means that the ratio of activity prior to and after the decontamination in radioactive water reached up to 275 . The potent activity is probably due to exchange between $\mathrm{Na}^{+}$and $\mathrm{Cs}^{+}$of monovalent cation. Eventually, the adsorbent is strongly recommended to be an excellent adsorbent for ${ }^{137} \mathrm{Cs}$. Figure 5 shows the experimental data and the curves fitted by the Langmuir model. It can be seen that the Langmuir model is suitable for the Cs adsorption isotherm one by magnetic Na-phlogopite adsorbent. The maximum adsorption capacity of the magnetic Na-phlogopite toward radioactive cesium $\left({ }^{137} \mathrm{Cs}\right)$ is determined to be $69.79 \mathrm{mg} / \mathrm{g}$. In environment, magnetic composites have attracted a lot of interest because of their distinguished properties that are a characteristic of there is no remanent magnetization when the magnetic field is removed.

\section{Conclusion}

In conclusion, we demonstrated the capability of magnetic composites to remove radioactive cesium in the environment. The potent magnetic composites were synthesized and characterized for their radioactive cesium adsorption properties with high removal efficiency. Furthermore, magnetic nanocomposites can be used as a rapid and ease technology for the removal of radioactive cesium in an environment using a magnetic field. Thus, the magnetic Na-phlogopite sorbent can offer high potential for in situ remediation.

Acknowledgments This work was supported by the National Research Foundation of Korea (NRF) grant funded by the Korea government (MSIP) (No. 2012M2A8A5025996). And, this work was in part supported by the National Research Council of Science \& Technology (NST), Korea.

\section{Compliance with ethical standards}

Conflict of interest The authors declare no competing financial interest.

\section{References}

Beveridge JS, Stephens JR, Williams ME (2011) The use of magnetic nanoparticles in analytical chemistry. Annu Rev Anal Chem 4:251-273

Buesseler KO, Jayne SR, Fisher NS, Rypina II, Baumann H, Baumann Z, Breier CF, Douglass EM, George J, Macdonald AM (2012) Fukushima-derived radionuclides in the ocean and biota off Japan. Proc Natl Acad Sci 109:5984-5988

Elliott DW, Zhang WX (2001) Field assessment of nanoscale bimetallic particles for groundwater treatment. Environ Sci Technol 35:4922-4926

Gleich B, Weizenecker J (2005) Tomographic imaging using the nonlinear response of magnetic particles. Nature 435:1214-1217

Grignard E, Guéguen Y, Grison S, Lobaccaro JM, Gourmelon P, Souidi M (2008) In vivo effects of chronic contamination with ${ }^{137}$ cesium on testicular and adrenal steroidogenesis. Arch Toxicol 82:583-589

Guéguen Y, Lestaevel P, Grandcolas L, Baudelin C, Grison S, Jourdain JR, Gourmelon P, Souidi M (2008) Chronic contamination of rats with ${ }^{137}$ cesium radionuclide: impact on the cardiovascular system. Cardiovasc Toxicol 8:33-40

Gupta RK, Dubey SS (2005) Removal of cesium ions from aqueous solution by polyaniline: a radiotracer study. J Polym Res $12: 31-35$

Gupta AK, Gupta M (2005) Synthesis and surface engineering of iron oxide nanoparticles for biomedical applications. Biomaterials 26:3995-4021

Hyeon T (2003) Chemical synthesis of magnetic nanoparticles. Chem Commun 8:927-934

Lu AH, Schmidt W, Matoussevitch N, Bönnemann H, Spliethoff B, Tesche B, Bill E, Kiefer W, Schüth F (2004) Nanoengineering of 
a magnetically separable hydrogenation catalyst. Angew Chem Int Ed Engl 43:4303-4306

Mahendran V, Philip J (2012) Nanofluid based optical sensor for rapid visual inspection of defects in ferromagnetic materials. Appl Phys Lett 100:073104

Mercer BW, Ames LL (1978) Zeolite ion exchange in radioactive and municipal waste treatment. In: Sand LB, Mumpton FA (eds) Natural zeolites: occurrence, properties and use. Pergamon, New York, pp 451-462

Sangvanich T, Sukwarotwat V, Wiacek RJ, Grudzien RM, Fryxell GE, Addleman RS, Timchalk C, Yantasee W (2010) Selective capture of cesium and thallium from natural waters and simulated wastes with copper ferrocyanide functionalized mesoporous silica. J Hazard Mater 182:225-231

Scott AD, Smith SJ (1966) Susceptibility of interlayer potassium in micas to exchange with sodium. Clays Clay Miner 14:69-81

Souidi M, Tissandie E, Grandcolas L, Grison S, Paquet F, Voisin P, Aigueperse J, Gourmelon P, Guéguen Y (2006) Chronic contamination with 137cesium in rat: effect on liver cholesterol metabolism. Int J Toxicol 25:493-497

Stout SA, Cho Y, Sridhar K (2006) Uptake of cesium and strontium cations by potassium-depleted phlogopite. Appl Clay Sci 31:306-313 